\title{
Perceptual differences in trail-following leaf-cutting ants relate to body size
}

\author{
C.J. Kleineidam ${ }^{\mathrm{a}, *}$, W. Rössler ${ }^{\mathrm{a}}$, B. Hölldobler ${ }^{\mathrm{a}, \mathrm{b}}$, F. Roces ${ }^{\mathrm{a}}$ \\ ${ }^{a}$ Department of Behavioral Physiology and Sociobiology, Biozentrum, University of Würzburg, Am Hubland, 97074 Würzburg, Germany \\ ${ }^{\mathrm{b}}$ School of Life Sciences, Center of Social Dynamics and Complexity, Arizona State University, Tempe, AZ, USA
}

\begin{abstract}
Leaf cutting ants of the genus Atta have highly size polymorphic workers, and size is related to division of labor. We studied trail following behavior of different sized workers in a laboratory colony of Atta vollenweideri. For small and large workers, we measured responsiveness and preference to artificial conspecific and heterospecific pheromone trails made from poison gland extracts of A. vollenweideri and A. sexdens. Responsiveness was measured as the probability of trail following, and preference was measured by testing the discrimination between one conspecific and one heterospecific trail. Minute amounts of the releaser component methyl 4 methylpyrrole 2 carboxylate $(0.4 \mathrm{pg} / 1 \mathrm{~m})$, present in both, conspecific and heterospecific trails, suffice to elicit trail following behavior. Workers followed heterospecific trails, and these trails (after normalizing their concentration) were as effective as conspecific trails. Small workers were less likely to follow a trail of a given concentration than large workers. In the discrimination test, small workers preferred the conspecific trail over the heterospecific trail, whereas large workers showed no significant preference. It is suggested that large workers primarily respond to the releaser component present in both trails, whereas small workers focus more on the conspecific traits provided by the blend of components contained in the trail pheromone.
\end{abstract}

Keywords: Chemical communication; Pheromone; Alloethism; Division of labor; Task allocation

\section{Introduction}

The worker caste in some social insect species is composed of individuals with a remarkable variation in body size and other morphological traits which is considered an adaptation that facilitates task allocation and division of labor (Wilson, 1971; Hölldobler and Wilson, 1990). The polyethism found in such colonies is known as alloethism, where size is related to the behavior of workers and their task allocation. Beside size-related task allocation, workers may be specialized by other morphological adaptations to specific tasks. For instance, in Solenopsis geminata, which collect seeds as part of their diet, large workers have mandibles shaped specifically for milling seeds (Wilson, 1978). The largest workers of

\footnotetext{
*Corresponding author. Tel.: + 49931 8884312; fax: + 499318884309.

E mail address: kleineidam@biozentrum.uni wuerzburg.de (C.J. Kleineidam).
}

polymorphic ant colonies often show morphological specialization for nest defense and are described as separate physical sub-caste, the soldiers, within the worker caste. For example, in the ant Camponotus (Colobopsis) truncatus large workers with a truncated head are able to block the nest entrance in response to threats, while the head shape of other workers prevents them from fulfilling this task (Forel, 1874). Thus, physical constraints may force workers to perform specific tasks and exclude them from others.

An important aspect of division of labor in social insects is its plasticity: in most cases, workers can switch between tasks and can change their responsiveness to task-allocating stimuli. Behavioral responsiveness is described by the probability of a worker to respond to task-associated stimuli. Division of labor is seen as an emergent property, based on qualitative and quantitative variations in individual responsiveness (Beshers and Fewell, 2001). In polymorphic colonies, responsiveness may be further 
influenced by interactions (communication) between workers as well as differences in the perception of taskassociated stimuli based on experience-dependent plasticity of the nervous system. Changing responsiveness of workers over time, a phenomenon known as age polyethism, adds further complexity to the colony organization of social insects.

The social organization of leaf-cutting ant colonies (genus Atta) is among the most complex found in all ants (Weber, 1972; Wilson, 1980; Fowler, 1983). Workers exhibit a 200 -fold variation in mass, which is one of the greatest known in ants, and the frequency distribution of different-sized workers is bimodal (Wilson, 1971; Oster and Wilson, 1978). Several studies describe differences in responsiveness to external stimuli in workers of different size. For example, small workers of Atta capiguara are disproportionately more likely to respond with an alarm reaction when exposed to crushed ant heads along foraging trails (Hughes and Goulson, 2001). Small workers appear to be more effective in fighting off conspecific intruders than large workers are (Salzemann and Jaffe, 1991). Although different colonies may forage harmoniously on the same foraging site, small workers are recruited massively when battles erupt between colonies (Salzemann and Jaffe, 1990; Whitehouse and Jaffe, 1996); and small workers of Atta sexdens show a higher trail fidelity than large workers (Morgan et al., 2006).

The mechanisms underlying the size-related recruitment in agonistic interactions are not understood. Size-related responsiveness of workers may be based on sensory limitations, such as receptor neuron numbers or sensitivity to an odor, leading to perceptual differences, or may be based on perceptual differences resulting from odor information processing in the brain when workers assess the trail chemistry. Latter perceptual differences, which could enable a colony to channel workers into different tasks on the trail, require plasticity of the nervous system. Indeed, developmental plasticity correlated with behavioral performance was found in the olfactory pathway of, e.g., honey bees (Tautz et al., 2003; Groh et al., 2004). In leafcutting ants, the first olfactory neuropils, the antennal lobes, contain one substantially enlarged glomerulus at the entrance of the antennal nerve. The enlarged glomerulus was found only in large workers; glomeruli in small workers are all similar in size, which indicates a developmental rather than an experience-dependent plasticity (Kleineidam et al., 2005). The antennal lobe design separates leaf-cutting ant workers into two distinct neuroanatomical castes based on the occurrence of one enlarged glomerulus (unpublished own data). This separation of workers according to their neuroanatomy suggests the possibility that small and large workers differ in how odor information is processed, and it probably results in different olfactory-guided behavior.

The aim of the present study was to investigate whether small and large leaf-cutting ant workers (Atta vollenweideri) differ in their response to different trail pheromones (conspecific vs. heterospecific trails), and whether sizerelated responsiveness may be based on perceptual differences.

For that aim, we first showed that $A$. vollenweideri workers also follow heterospecific $(A$. sexdens) trails, and respond in a concentration-dependent manner. Then, both small and large workers were tested whether they discriminate between two competing trails (conspecific vs. heterospecific), using pheromone concentrations that were normalized according to the trail-following response of workers on single trails. This normalization was used to assess perceptual differences based on odor information processing of workers of different size and to compensate for their size-related sensory differences.

\section{Materials and methods}

\subsection{Trail pheromone system of Atta and artificial trails}

The trail pheromone of Atta is a blend of many components, and two of them (methyl-4-methylpyrrole-2carboxylate (M4MP2C) and 2-ethyl-3,6-dimethylpyrazine $(2 \mathrm{E} 3,6 \mathrm{DMP}))$ are found in relatively high quantities (main pheromone components) in the secretions of the poison gland. The ratio of both defines the species-specificity with the releaser component (sufficient to elicit trail-following behavior) in highest quantity in the poison gland content (Cross et al., 1979; Evershed and Morgan, 1983; Billen et al., 1992). The poison gland components of $A$. vollenweideri were so far unknown. We provide behavioral evidence demonstrating that $\mathrm{M} 4 \mathrm{MP} 2 \mathrm{C}$ is the releaser component of A. vollenweideri (Section 3). 2E3,6DMP alone does not release trail-following behavior. M4MP2C is the releaser component of all investigated Atta species with the exception of $A$. sexdens. The releaser component of $A$. sexdens is 2E3,6DMP which, in this species, is present in higher quantity in the poison gland secretions than M4MP2C (14:1; Evershed and Morgan, 1983). Thus, $A$. sexdens poison glands contain the releaser component of other Atta species including A. vollenweideri. The releaser component of $A$. sexdens (2E3,6DMP) does not elicit trail-following behavior in several other Atta species when presented alone. In natural trails, the releaser component is combined with other components of the poison gland contents. It has been suggested that this blend of components underlies heterospecific trail-following in leaf-cutting ants (Mintzer, 1980; Morgan et al., 2006).

For the experimental trails, we used two synthetic components (the two releaser components M4MP2C (Sigma-Aldrich, USA) and 2E3,6DMP and its isomer 2E3,5DMP at a ratio of 1:1 (Oxford Chemicals, UK)) of the Atta trail pheromone, and poison gland extracts of A. vollenweideri and $A$. sexdens workers.

The concentrations of the synthetic components in the stock solutions were measured after the experiments by capillary gas chromatography (GC). GC analyses were performed on a HP 5890 II GC (Hewlett-Packard, 
Avondale, PA) with a flame ionization detector (FID) and a "cool on column" injection using hydrogen as carrier gas. For GC conditions, see Kleineidam et al. (2005). The concentration of M4MP2C in the stock solution was $2.45 \mathrm{mg} / \mathrm{ml}$. The concentration of $2 \mathrm{E} 3,6 \mathrm{DMP}$ in the stock solution was $14.45 \mathrm{mg} / \mathrm{ml}$.

The stock solutions of the gland extracts were obtained from poison glands of 10 workers diluted in $1 \mathrm{ml}$ of hexane. The gland extract of $A$. vollenweideri workers (AVG; for conspecific trails) was obtained from large workers of the same colony later tested in the behavioral experiments. The gland extract of Atta sexdens rubropilosa workers (ASG; for heterospecific trails) was obtained from a laboratory colony reared under similar conditions as the $A$. vollenweideri colony.

\subsection{Animals}

The $A$. vollenweideri (Forel) colony was reared at the Biozentrum, University of Würzburg, in an environmental chamber at $25^{\circ} \mathrm{C}$ and $50 \%$ relative humidity in a $12 \mathrm{~h} / 12 \mathrm{~h}$ photoperiod and fed mainly with privet leaves (Ligustrum vulgaris) and dog rose (Rosa canina). The colony was collected in 2002 in El Bagual, Formosa, Argentina (Bollazzi and O. Geissler). At the time of the experiments, the fungus garden occupied a volume of about 121 in six plastic boxes $(19 \times 19 \times 9 \mathrm{~cm})$ interconnected with plastic tubes. The A. sexdens colony was collected in 1993 in Botucatu, Brazil (L.C. Forti). It was of similar size and was treated like $A$. vollenweideri regarding environmental and feeding conditions.

\subsection{Classification of small and large workers}

After the behavioral experiments, each tested worker's body size was measured. Head width (HW; distance between the outer edges of both eyes) or body mass (BM) was used as a measure of body size. HW and BM were highly correlated in 280 workers for which both measurements were taken $\left(\mathrm{HW}=\mathrm{BM}^{0.3672}-0.0443\right.$; $\left.r^{2}=0.96 ; p<0.01\right)$. The BM distribution of foraging workers was measured for 500 individuals during one foraging bout, and the size distribution was calculated (Fig. 1). The size distribution found is unimodal and slightly skewed to the right. Only $15 \%$ of all workers were small workers during this time of the foraging bout.

Workers with a BM of more than $2.78 \mathrm{mg}$ or a $\mathrm{HW}$ of more than $1.4 \mathrm{~mm}$ were considered as large workers. This classification was used because workers smaller than $1.4 \mathrm{~mm}$ HW were never seen transporting leaf fragments on the foraging bridge.

\subsection{Experimental setup}

During experiments, the colony had free access to a foraging arena $(40 \times 60 \mathrm{~cm})$ via a wooden bridge $(60 \mathrm{~cm})$. At the foraging arena, the colony was offered fresh dog

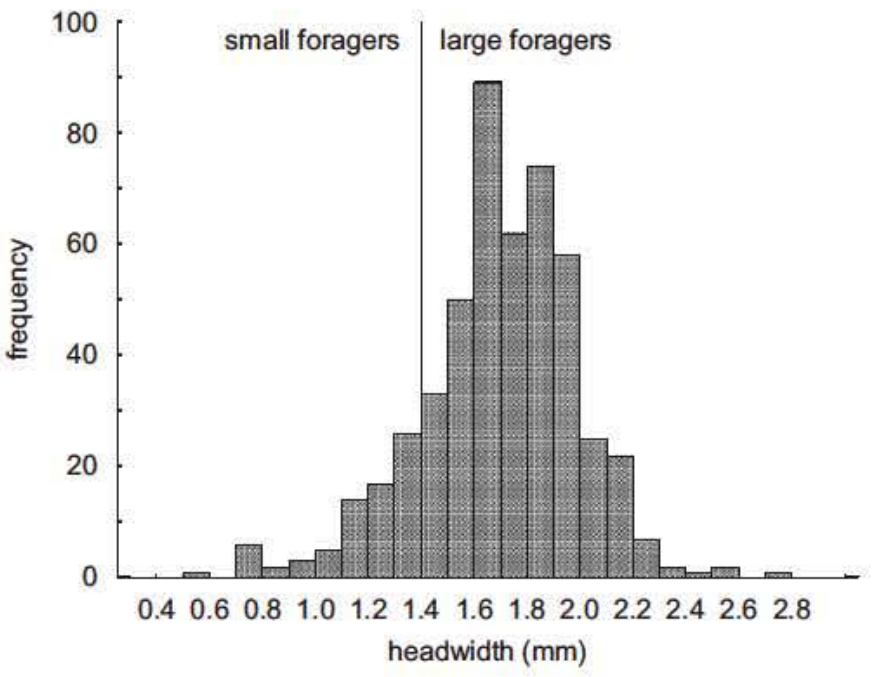

Fig. 1. Size distribution of foragers on the wooden bridge (trail) and in the foraging arena. Body mass of 500 foragers was measured and the corresponding head width was calculated based on a previously obtained relationship. Foragers were separated into two groups, small workers with a head width of $\leqslant 1.4 \mathrm{~mm}$ or a body mass of $\leqslant 2.78 \mathrm{mg}$ and large foragers with sizes above. Small foragers accounted for $15 \%$ of all foragers at the end of this foraging bout.

rose ( $R$. canina) leaves, which were cut by the workers into fragments and carried across the bridge to the nest. On their way towards the nest, single workers were branched off the bridge onto a short bridge guiding them to a paper platform. On the paper platform, the workers were tested for trail-following on artificially laid trails.

The artificial trails were applied with a microliter syringe $(10 \mu \mathrm{l})$ onto a printed-Y on a paper platform (laser print out on regular copy paper). Each trail covered a total length of $6 \mathrm{~cm}$ along the base and one arm of the printed-Y. Along the base and the other arm we applied either the solvent as control or $10 \mu \mathrm{l}$ of the alternative trail pheromones (gland extract) as competing trail. The solvent (hexane) was allowed to evaporate for $10 \mathrm{~min}$, prior to the behavioral experiments. Workers were considered to follow a trail when they clearly moved along the trail for at least $25 \mathrm{~mm}$ on one arm of the printed-Y. Note, since the workers could cross the trails and move freely on the paper platform, this test-design for trail-following behavior is not a binary-choice experiment.

The workers responsiveness was calculated as the percentage of workers following a particular trail. Thus, responsiveness corresponds to the probability of a worker (of a particular group) to follow a trail.

\subsection{Responsiveness on single trails}

The experiments on single trails were used: (i) to identify which of the two known main trail pheromone components is the releaser component in A. vollenweideri. In the course of identification, we determined the lowest quantity of the behaviorally identified releaser component that still elicits trail-following behavior in half of the tested large workers; 
(ii) to determine the lowest quantity (concentration) of the two gland extracts AVG and ASG necessary to elicit trail-following behavior in half of the (large) workers (responsiveness of $50 \%$ ); and (iii) to investigate whether artificially laid heterospecific trails (ASG trails) presented at higher concentration can elicit trail-following behavior in most $A$. vollenweideri workers and thus can be used as a potentially effective trail signal in our experiments.

Successively, 20 workers were tested individually in the first trial before the platform was exchanged, and in all following trials the side with the pheromone trail was alternated. No trail laying was observed in the workers entering the paper platform, and the paper platform did not show any visual signs of marking by workers after the experiments.

Since the worker's size was measured after the experiments, the number of small and large workers differed in each trial. We analyzed only those trials in which at least five workers in each group (small and large workers) were tested. This was done to consecutively allow the analysis of differences in responsiveness between small and large workers. Ten trials were performed at the lowest concentration and six trials at each of two higher concentrations of M4MP2C, AVG and ASG trails, resulting in a total of 66 trials (1320 workers tested). 2E3,6DMP trails were offered at five different concentrations (six trials), and a total of 120 workers were tested. Since very few workers followed these trails, the responsiveness was not analyzed any further.

The significance of differences in responsiveness of workers on M4MP2C, AVG and ASG trails at the three different concentrations was tested using the KruskalWallis ANOVA. The significance of difference in responsiveness of small vs. large workers was tested across all concentrations used, but separately for M4MP2C, AVG and ASG trails with the Wilcoxon matched pair test.

\subsection{Responsiveness and preference on competing trails}

The experiments on competing trails were used to measure the preference of small and large workers to AVG trails vs. ASG trails. The idea of the experiment was that the releaser component on the heterospecific trail, provided the concentration is high enough, may compensate for any missing cues present on the conspecific trail. If this is the case, the responsiveness of, e.g., large workers to follow either trail should be balanced and perceptual differences between small and large workers in assessing the trails can possibly be measured.

Competing trails of AVG and ASG were simultaneously presented along the base and separately along one arm of the printed-Y. The paper platform was exchanged after each worker tested, and the two pheromone trails were switched after each trial to avoid side biases. A total of 320 workers were tested on competing AVG and ASG trails.
In the first experiment, 160 workers (58 small and 102 large workers) were tested on competing trails with the AVG trails at a stock dilution of $10^{-6}$ and the ASG trails at a stock dilution of $10^{-5}$. The concentrations used were balanced (normalized), based on the results from the experiments with workers on single trails where workers responsiveness was similar and low $(51 \%$ of the large workers and $44 \%$ of all workers).

In the second experiment, again 160 workers (70 small and 90 large workers) were tested on AVG trails at a stock dilution of $10^{-6}$ and ASG trails at a stock dilution of $10^{-4}$. Thus, in the second experiment, the conspecific trail was equal compared to the first experiment, and the heterospecific trail was increased 10 -fold. Since workers showed a high responsiveness on single ASG trails at high concentration, the preference to the conspecific trail was expected to be reduced in this situation.

The responsiveness of both, small and large workers (in this case the percentage of workers following any of the two competing trails), was compared using a two by two contingency table and the Fisher's exact $p$-test. The trail preference of both, responsive small and large workers was also compared using a two by two contingency table and the Fisher's exact $p$-test. All statistical analyses were performed using the statistic software STATISTICA 7.1 (StatSoft, Oklahoma).

\section{Results}

\subsection{Releaser component of $A$. vollenweideri}

Workers of the leaf-cutting ant A. vollenweideri follow artificially laid trails with synthetic M4MP2C at very low concentration. Trails with a loading of $24.5 \mathrm{fg}$ M4MP2C over a length of $6 \mathrm{~cm}\left(10 \mu \mathrm{l}\right.$ stock dilution $\left.\times 10^{-9}\right)$ are sufficient to elicit trail-following behavior in 101 of 200 workers (10 trials with 20 workers each), and results in a median responsiveness of $45 \%$ (Fig. 2). Higher concentrations of the synthetic trail component M4MP2C led to a massive increase in responsiveness. Significantly, more workers followed the M4MP2C trail when the concentration was increased to a loading of $2.45 \mathrm{pg}$ (100-fold) and $245 \mathrm{pg}(10,000$-fold), with a responsiveness averaging $85 \%$ (Kruskal-Wallis ANOVA: $H(2, N=22)=15.03$, and both $p<0.01)$.

No pronounced trail-following behavior was observed on artificial trails with 2E3,6DMP. When presented together with its synthetic isomer, 2E3,6DMP elicits trailfollowing in only one to three workers, according to our measure of trail-following, in any of the six different trials. This corresponds to a responsiveness of $\max 15 \%$ at any of the five different concentrations tested (loading of $1.45 \mathrm{fg}-144.5 \mathrm{ng})$.

From the high responsiveness of workers to trails laid with the synthetic M4MP2C, we conclude that M4MP2C is the releaser component of the trail pheromone in A. vollenweideri. 


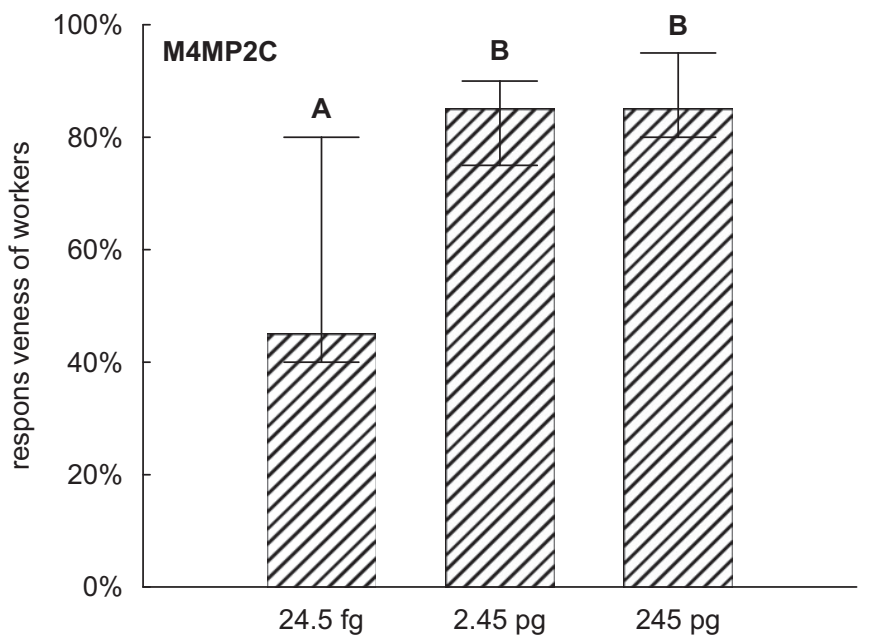

Fig. 2. Responsiveness of workers on M4MP2C trails at different concentrations. At a loading of $24.5 \mathrm{fg}$ M4MP2C onto the printed $\mathrm{Y}$. Two hundred workers were tested in 10 trials; at the two higher concentrations, six trials each were performed, resulting in a total of 440 workers tested. Bars indicate the median and whiskers the range of the percentage of workers following the M4MP2C trail. Differences in responsiveness were tested with the Kruskal Wallis ANOVA, and statistically significant differences at the $p<0.01$ level are indicated by different letters.

\subsection{Responsiveness of workers to single $A V G$ and $A S G$ trails}

In preliminary experiments, various concentrations of the gland extracts were tested in order to estimate the minimal concentration necessary to elicit trail-following in about half of the workers. A dilution of $10^{-6}$ of the AVG stock solution and $10^{-5}$ of the ASG stock solution were selected for further experiments. In order to quantify the responsiveness of workers at high gland extract concentrations, we used concentrations 100 and 10,000 times higher than the lowest concentration used.

At the lowest concentration used, the responsiveness of workers was $45 \%$ and $43 \%$ on AVG and ASG trails, respectively (Fig. 3). At higher concentrations of both AVG and ASG trails, the responsiveness of workers increased. At the highest concentration used, responsiveness of workers was higher than $80 \%$ and significantly different from that at the lowest concentration, both for AVG and ASG (Fig. 3, Kruskal-Wallis ANOVA: $H$ $(2, N=22)=16.29$ and 15.04 , respectively, and both $p<0.01)$. Thus, an heterospecific trail can be as effective as a conspecific trail when presented at a higher concentration, inducing high responsiveness of workers. Moreover, the range of measured concentration-dependent responsiveness is similar on both AVG and ASG trails.

\subsection{Difference in responsiveness of small and large workers on single trails}

Workers were scored into small and large workers after the experiments. In general, for small workers a lower
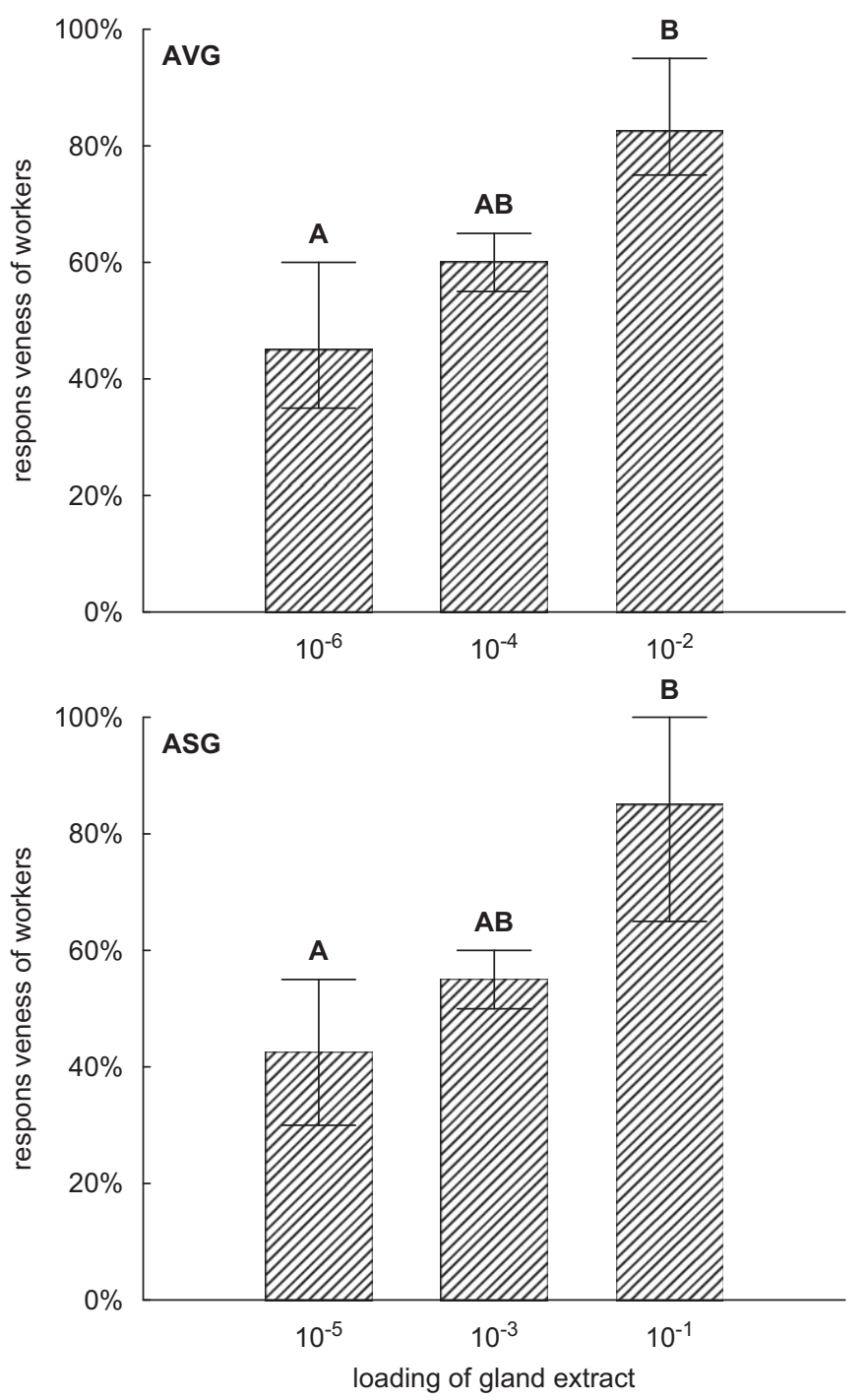

Fig. 3. Responsiveness of workers on AVG trails (AVG) and ASG trails (ASG) at different concentrations. On the $X$ axis, the different dilutions of the stock solutions used are indicated. Bars indicate the median and whiskers the range of the percentage of workers following the AVG and ASG trails. Differences in responsiveness were tested with the Kruskal Wallis ANOVA, and statistically significant differences at the $p<0.01$ level are indicated by different letters.

responsiveness was measured on AVG and ASG trails than for large workers across all concentrations (Wilcoxon matched pair test: AVG, difference of median $=18 \%, n=22$ trials, $Z=2.42, p<0.05 ;$ ASG, difference of median $=22 \%, n=22$ trials, $Z=2.24$, $p<0.05)$.

At the lowest concentration of AVG trails, the responsiveness of small workers was $40 \%$ and did not differ significantly from the $52 \%$ of responsive large workers (Wilcoxon matched pair test: $n=10$ trials, $Z=1.48$, $p \geqslant 0.05)$. At the lowest concentration of ASG trails, the responsiveness of small workers was $33 \%$ and differed significantly from the $50 \%$ of responsive large workers (Wilcoxon matched pair test: $n=10$ trials, $Z=2.50$, $p<0.05)$. The found difference in responsiveness between 
small and large workers to the heterospecific trail (ASG trail) but not to conspecific trail (AVG trail) indicates that workers may differ in how they evaluate the trails. This was tested in the following experiment with competing trails.

\subsection{Responsiveness and preference on competing trails}

Two experiments with competing trails presented simultaneously on the printed-Y on the paper platform were performed. In the first experiment (C1), the conspecific (AVG) trail and the heterospecific (ASG) trail were at a normalized concentration according to the trail-following response of large workers on single trails $52 \%$ on AVG trails at $10^{-6}$ vs. $50 \%$ on ASG trails at $10^{-5}$ ). In the second experiment (C2), the heterospecific (ASG) trail was presented at a higher concentration (AVG $10^{-6}$ vs. ASG $10^{-4}$ ).

The probability of following either the AVG trail or the ASG trail (responsiveness) was greater than that observed for workers following single trails at corresponding concentrations. In addition, the probability of trailfollowing was similar for small and large workers. In experiment $\mathrm{C} 1,71 \%$ of the small workers $(n=58)$ and $76 \%$ of the large workers $(n=102)$, and in experiment $\mathrm{C} 2$, $67 \%$ of small workers $(n=70)$ and $64 \%$ of large workers $(n=90)$ followed one of the competing trails. No significant difference between small and large workers was found in both experiments (Fisher's exact $p$-test: C1, $p=0.49$ and $\mathrm{C} 2, p=0.43$ ). The similar responsiveness of small and large workers on competing trails indicates that both trails together are similarly attractive to small and large workers.

The competing trails forced workers to discriminate between two trails (discrimination test), and to decide to follow one of them. Size-related trail preference was calculated as the percentage of workers following the AVG trail, taking into account the total number of workers showing trail-following (responsive workers). Small and large workers differed in their preference (Fig. 4). In experiment $\mathrm{Cl}$, the responsive small workers preferred the AVG trail $(73 \%)$ over the ASG trail, compared to large workers, which in the same situation followed both trails with equal probability ( $51 \%$ followed the AVG trail). The difference between small and large workers in this experiment was statistically significant (Fisher's exact $p$ test: $p<0.05$ ) (Fig. 4, left). In experiment $\mathrm{C} 2$, in which the heterospecific trail was presented at a higher concentration (one order of magnitude), the responsive small workers followed both trails with equal probability ( $53 \%$ followed the AVG trail) and the large workers tended, although not statistically significant, to prefer the ASG trail $(59 \%$ followed the ASG trail). However, the difference between small and large workers in experiment $\mathrm{C} 2$ was not significantly different (Fisher's exact $p$-test: $p=0.16$ ) (Fig. 4, right).

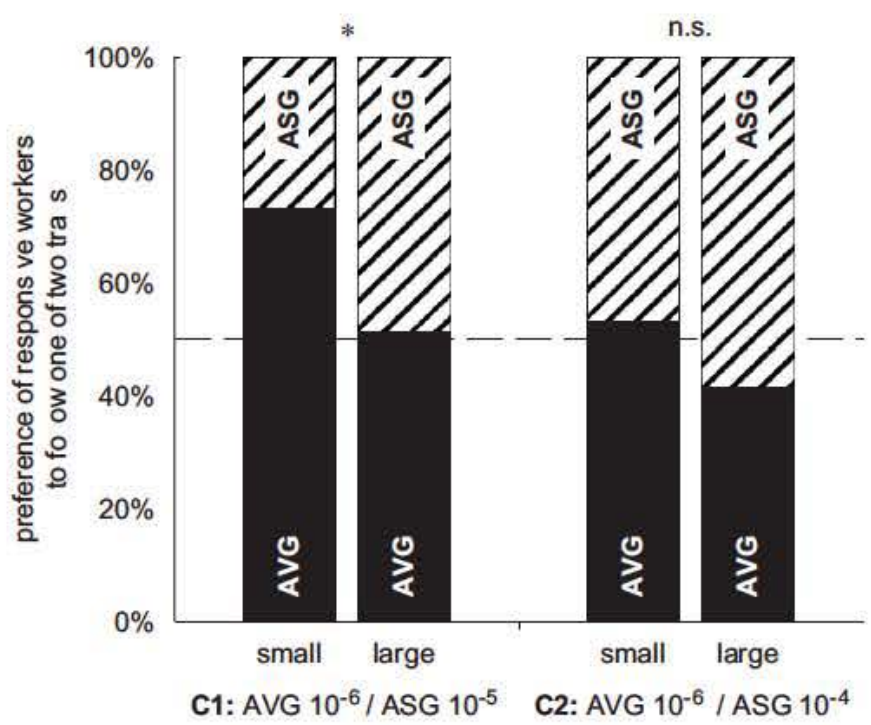

Fig. 4. Workers' preference on competing trails. The percentages of small and large workers following the AVG trail (black bars) were compared in two experiments ( $\mathrm{Cl}$ and $\mathrm{C} 2$ ). The remaining responsive workers (hatched bars) followed the ASG trail. In experiment C1 (AVG $10^{6}$ vs. ASG $10{ }^{5}$ ), the responsive small workers preferred the AVG trail over the ASG trail compared to large workers. In experiment C2 (AVG $10^{6} \mathrm{vs.} \mathrm{ASG} 10^{4}$ ), the responsive small workers followed both trails with equal probability, and the large workers tended to prefer the ASG trail. The responsive small workers and the responsive large workers were compared using the Fisher's exact $p$ test and significant differences $(p<0.05)$ are indicated by an asterisk.

\section{Discussion}

Workers of the leaf-cutting ant $A$. vollenweideri are able to detect and discriminate heterospecific trails of $A$. sexdens from conspecific trails. However, small and large workers differ in how they assess conspecific and heterospecific trails when confronted in a discrimination test. We suggest that large workers primarily respond to the releaser component present in both conspecific and heterospecific trails, whereas small workers focus more on the conspecific cues provided by the blend of components contained in the trail pheromone.

\subsection{Sensitivity to the releaser component of the trail pheromone}

Our experiments with the two synthetic main components of the Atta trail pheromone provide strong evidence that M4MP2C is the most important substance in the poison gland of $A$. vollenweideri since it functions as releaser component by eliciting trail-following behavior. Workers of $A$. vollenweideri follow trails drawn with minute amounts of M4MP2C. For a distance of $1 \mathrm{~m}$, a calculated amount of only $0.4 \mathrm{pg}$ of M4MP2C suffices to induce trail-following behavior in $50 \%$ of large workers. Thus, a much lower quantity of the releaser component is sufficient for eliciting trail-following than that described for other Atta species, e.g. Atta texana or Atta cephalotes 
(Riley et al., 1974; Robinson et al., 1974). Therefore, an often cited calculation that $1 \mathrm{mg}$ of the releaser component M4MP2C, if deposited with maximal efficiency, would suffice to induce trail-following in ants three times around our planet (Tumlinson et al., 1971), is a striking underestimation for $A$. vollenweideri. Although these estimations are based on trail-following behavior in a non-native setting and with artificially laid trails, they illustrate the amazing pheromone sensitivity of ants.

M4MP2C from the poison gland of the leaf-cutting ant A. texana is the first chemically identified trail pheromone in ants (Tumlinson et al., 1971, 1972). Since then, a number of studies demonstrated the function of M4MP2C as releaser component of the trail pheromone in other leafcutting ant species, and additional compounds of the poison gland secretion in various species of the genus Atta have been identified (Moser and Silverstein, 1967; Riley et al., 1974; Evershed and Morgan, 1983). So far, only in $A$. sexdens a different releaser component 2E3,6DMP has been identified. In $A$. vollenweideri, we found no trailfollowing behavior on trails drawn with 2E3,6DMP and at high concentrations some workers showed in fact alarm behavior by elevating their bodies and gaping their mandibles (data not shown).

\subsection{Specificity of the trail pheromone}

Leaf-cutting ants do show heterospecific trail-following behavior (Mintzer, 1980), and the ratio of the two main components (M4MP2C and 2E3,6DMP) provides speciesspecificity in some cases (Robinson et al., 1974; Billen et al., 1992). Our study shows heterospecific trail-following by workers of $A$. vollenweideri. Workers follow trails made of poison gland extract of $A$. sexdens (ASG trails). The ratio of the two main components in the poison gland secretion of $A$. sexdens is about $14: 1$, with the releaser component 2ED3,6DMP in higher quantity, and a mixture of both components proved to be more potent in behavioral assays than 2ED3,6DMP alone (Evershed and Morgan, 1983; Billen et al., 1992).

Besides providing species specificity, multi-component pheromones like the trail pheromone of Atta with its two main components, seem to widen the concentration range within which workers may respond, thus leading to graded colony response. Our results indicate that at the colony level, response thresholds are more variable when multicomponent signals (AVG or ASG trails) are presented compared to a presentation of a single component signal (M4MP2C trails). We observed that for M4MP2C, and gland extracts, the responsiveness of workers increased with increasing pheromone concentration on the trails, as was shown also, e.g. A. sexdens (Morgan et al., 2006). On M4MP2C trails, the responsiveness increased significantly after a 100-fold increase in concentration (Fig. 3). On AVG and ASG trails, on the other hand, the responsiveness of workers increased gradually with increasing concentrations on the trails, and the highest responsiveness occurred after a 10,000-fold increase in concentration. On M4MP2C trails the responsiveness of workers probably also increases gradually but within a smaller concentration range, and was therefore not detected by our behavioral assay. It seems that the releaser component (M4MP2C) triggers trail-following behavior of workers within a small concentration range when presented alone.

The measured concentration-dependent responsiveness was similar on both single AVG and single ASG trails, and the high responsiveness of workers on ASG trails shows that heterospecific trails can be as effective as conspecific trails, provided, the concentration is high enough. Thus, worker's responsiveness to the heterospecific trail is not a constraint in the discrimination test. This is important, since it is a necessary condition for measuring perceptual preferences independent of sensory or morphological limitations of the workers tested in the experiments with competing trails.

\subsection{Normalized concentrations of $A V G$ and $A S G$ trails}

Trail-following as a behavioral response requires both the detection of at least one component of the pheromone, and the recognition of the stimulus as behaviorally relevant. Detection and recognition cannot be separated experimentally because recognition is necessary for the observed behavior (trail-following) and implies detection. In order to reduce differences between small and large workers in detecting different trails, we selected concentrations which elicit similar responsiveness (normalized concentrations of AVG and ASG). At the normalized concentrations in our experiments, the concentration of the releaser component (M4MP2C) on the heterospecific (ASG) trail might either be same or lower (i), or higher (ii) than on the conspecific trail. If (i) the concentration of the releaser component is same or lower, then there have to be components on the ASG trail which are even more behaviorally relevant than the conspecific components on the AVG trail. This is a highly unlikely possibility, because then an ant-laid trail of $A$. sexdens would be at least as potent in recruiting $A$. vollenweideri workers as an ant-laid trail of A. vollenweideri. However, we have not tested how potent natural $A$. sexdens trails are in recruiting $A$. vollenweider workers compared to conspecific trails. If (ii) the concentration of the releaser component is higher, then the A. sexdens specific components (or ratios) may have no impact on the responsiveness, or might reduce the responsiveness of the $A$. vollenweideri workers. In this case, the concentration of a releaser component must compensate for the effects of the missing A. vollenweideri specific components (or ratios) on ASG trails.

This general consideration of the trail pheromone communication in Atta together with our results supports the assumption that $A$. vollenweideri uses M4MP2C as releaser component and that this component is at a higher concentration on ASG trails than on AVG trails, when both are presented at a normalized concentration. 


\subsection{The behavior of small vs. large workers}

On single conspecific and heterospecific trails, the responsiveness of small workers was lower than the responsiveness of large workers. Are large workers more sensitive than small workers? Our experiments were not designed to detect the absolute sensitivity of the workers to the gland extracts. Not only potential differences in sensory "equipment" but also physical constraints may interact with the ants' behavioral performance, e.g. size-related differences in search patterns or step size along the pheromone trail. Such size-related constraints may also interact with our measure of responsiveness. However, it may well be that large workers are more sensitive to the pheromone and its components than small workers. Large workers are assumed to detect odors at lower concentrations than small workers do because of the larger number of sensilla and associated olfactory receptor neurons on their antennae. In other sensory modalities, e.g. sensing vibrations, physical differences may favor higher sensitivity in small workers. In fact, small workers of $A$. cephalotes are several times more sensitive than large workers to substrate-borne vibrations measured by multi-unit responses from the leg nerve (Markl, 1970).

On competing conspecific and heterospecific trails, both at low concentration, small and large workers showed a similar and high responsiveness, probably due to the twofold trail (AVG and ASG trail) at the printed-Y prior to its bifurcation. Small workers showed discrimination and preferred the conspecific trail (AVG) over the heterospecific trail (ASG), whereas in the same situation, large workers showed no preference for one of them. Do small workers indeed select the conspecific trail or is the shown preference the result of small workers' inability to detect the heterospecific trail? On single conspecific and single heterospecific trails both at a low concentration, the responsiveness of small workers was lower on the heterospecific trail than on the conspecific trail (43\% vs. $45 \%)$. Referring to the reasoning elaborated above, the releaser component is most probably at a higher concentration on ASG trails than on AVG trails. Thus, at least as many small workers should be able to detect the releaser component on ASG trails as those on AVG trails. The lower responsiveness of small workers on ASG trails is probably caused by missing conspecific cues that enhance the responsiveness on AVG trails or by components in the ASG reducing responsiveness (repellent substances). The latter is improbable because of the high responsiveness of A. vollenweideri workers on ASG trails at high concentrations (experiment C2).

We suggest that the releaser component M4MP2C and its putative higher quantity on the heterospecific trail represent the salient stimulus for large workers, and it therefore overrides heterospecific cues contained in the ASG trail. However, this was not the case for small workers preferring the conspecific trail. The fact that increasing the concentration of the heterospecific trail diminished the preference of small workers for the conspecific trail further supports our idea. It seems that when assessing competing trails at low pheromone concentration, trail-following in small workers is governed more by species-specific cues, whereas in large workers, the releaser component dominates all other trail pheromone traits. Such size-related assessment would require differences between small and large workers in the way they process odor information. For A. vollenweideri workers, size-related differences in the neuroanatomical design of the first olfactory neuropil are known, and these may underlie the distinct behavioral performance found in this study (Kleineidam et al., 2005).

\subsection{What is the biological significance of alloethism in trail discrimination?}

Our behavioral analysis is based on experiments with workers of a laboratory colony foraging in a non-native environment that allowed good control of trail quality and concentrations. However, the natural environment is much more complex, and the ultimate explanations for alloethism in trail discrimination remain to be investigated. At this point, we can only speculate about several possible scenarios.

At the individual level, loosing the trail might be fatal for a small worker, while a large worker, with its larger exploration range, might find their way back to the trail. The large workers are the ones that search for suitable food plants so that detecting incipient trails with minute amounts of the releaser component might be advantageous. The different responses of small and large workers to incipient trails may lead to a more precise allocation of small workers to different tasks along the conspecific trail. Trails are mainly used in the context of foraging and nest relocation, but also for recruitment to a threat along the trail or at the feeding site. Relatively more small ants are recruited to a threat caused by conspecific or heterospecific ants than to a threat simulating a vertebrate disturbance (Salzemann and Jaffe, 1991; Whitehouse and Jaffe, 1996). In addition, it has been reported that leaf-cutting ants show territorial behavior to defend resources and employ Dufour gland secretions as a territorial marker along the trunk trails (Salzemann et al., 1992). Thus, workers encounter a large variety of complex communication signals along the trails and small workers may be adapted for a more flexible response to trail signals.

\section{Acknowledgments}

We thank Kerstin Schmitter, Stefan Harth and Martin Schmid for experimental support, and two anonymous reviewers for their helpful comments on the manuscript. We are grateful that we were allowed to collect colonies of A. vollenweideri at the Estancia El Bagual and we greatly appreciate our stay at the biological field station of the Reserva Ecológica El Bagual (Formosa, Argentina). We also thank M. Bollazzi, O. Geissler (Würzburg) and 
L. Forti (Botucatu, Brazil) for collecting the A. vollenweideri and $A$. sexdens colonies. This work was funded by the Deutsche Forschungsgemeinschaft (DFG) and SFB 554 (A6 and E1).

\section{References}

Beshers, S.N., Fewell, J.H., 2001. Models of division of labor in social insects. Annual Review of Entomology 46, 413440.

Billen, J., Beeckman, W., Morgan, E.D., 1992. Active trail pheromone compounds and trail following in the ant Atta sexdens sexdens (Hymenoptera: Formicidae). Ethology Ecology \& Evolution 4, 197202.

Cross, J.H., Byler, R.C., Ravid, U., Silverstein, R.M., Robinson, S.W., Baker, P.M., Sabino de Oliveira, J., Jutsum, A.R., Cherrett, M.J., 1979. The major component of the trail pheromone of the leaf cutting ant, Atta sexdens rubropilosa Forel. 3 ethyl 2,5 dimethylpyrazine. Journal of Chemical Ecology 5, 187203.

Evershed, R.P., Morgan, E.D., 1983. The amounts of trail pheromone substances in the venom of workers of four species of attine ants. Insect Biochemistry 13, 469474.

Forel, A., 1874. Les fourmis de la Suisse. Systématique, notices anatomiques et physiologiques, architecture, distribution géographi que, nouvelles expériences et observations de moeurs. Neue Denks chriften der Allgemeinen Schweizerischen Gesellschaft fur die Gesammten Naturwissenschaften 26, 1452.

Fowler, H.G., 1983. Alloethism in a leaf cutting ant: laboratory studies on Atta texana (Hymenoptera: Formicidae: Attini). Zoologische Jahrbu cher, Abteilung fur Allgemeine Zoologie und Physiologie der Tiere 87, 529538.

Groh, C., Tautz, J., Rossler, W., 2004. Synaptic organization in the adult honey bee brain is influenced by brood temperature control during pupal development. Proceedings of the National Academy of Sciences of the United States of America 101, 42684273.

Holldobler, B., Wilson, E.O., 1990. The Ants. Harvard University Press, Cambridge, MA.

Hughes, W.O.H., Goulson, D., 2001. Polyethism and the importance of context in the alarm reaction of the grass cutting ant, Atta capiguara. Behavioral Ecology and Sociobiology 49, 503508.

Kleineidam, C.J., Obermayer, M., Halbich, W., Rossler, W., 2005. A macroglomerulus in the antennal lobe of leaf cutting ant workers and its possible functional significance. Chemical Senses 30, 383392.

Markl, H., 1970. Communication by stridulatory signals in leaf cutting ants. 3. Sensitivity to substrate vibrations. Zeitschrift fur vergleichende Physiologie 69, 6.

Mintzer, A., 1980. Simultaneous use of a foraging trail by two leafcutter ant species in the Sonoran Desert. Journal of the New York Entomological Society 88, 102105.
Morgan, E.D., Keegans, S.J., Tits, J., Wenseleers, T., Billen, J., 2006. Preferences and differences in the trail pheromone of the leaf cutting ant Atta sexdens sexdens (Hymenoptera: Formicidae). European Journal of Entomology 103, 553558.

Moser, J.C., Silverstein, R.M., 1967. Volatility of trail marking substance of the town ant. Nature 215, 206207.

Oster, G.F., Wilson, E.O., 1978. Caste and Ecology in the Social Insects. Princeton University Press, Princeton, NJ.

Riley, R.G., Silverstein, R.M., Carroll, B., Carroll, R., 1974. Methyl 4 methylpyrrole 2 carboxylate: a volatile trail pheromone from the leaf cutting ant, Atta cephalotes. Journal of Insect Physiology 20, 651654.

Robinson, S.W., Moser, J.C., Blum, M.S., Amante, E., 1974. Laboratory investigations of the trail following responses of four species of leaf cutting ants with notes on the specificity of a trail pheromone of Atta texana (Buckley). Insectes Sociaux 21, 8794.

Salzemann, A., Jaffe, K., 1990. On the territorial behavior of field colonies of the leaf cutting ant Atta laevigata (Hymenoptera, Myrmicinae). Journal of Insect Physiology 36, 133138.

Salzemann, A., Jaffe, K., 1991. Polyethism and nest defense in the leaf cutting ant Atta laevigata (Smith, F.). Insectes Sociaux 38, 149159.

Salzemann, A., Nagnan, P., Tellier, F., Jaffe, K., 1992. Leaf cutting ant Atta laevigata (Formicidae, Attini) marks its territory with colony specific Dufour gland secretion. Journal of Chemical Ecology 18, 183196.

Tautz, J., Maier, S., Groh, C., Rossler, W., Brockmann, A., 2003. Behavioral performance in adult honey bees is influenced by the temperature experienced during their pupal development. Proceedings of the National Academy of Sciences of the United States of America $100,73437347$.

Tumlinson, J.H., Silverstein, R.M., Moser, J.C., Brownlee, R.G., Ruth, J.M., 1971. Identification of the trail pheromone of a leaf cutting ant, Atta texana. Nature 234, 348349.

Tumlinson, J.H., Moser, J.C., Silverstein, R.M., Brownlee, R.G., Ruth, J.M., 1972. A volatile trail pheromone of the leaf cutting ant, Atta texana. Journal of Insect Physiology 18, 809814.

Weber, N.A., 1972. Gardening Ants the Attines. The American Philosophical Society, Philadelphia.

Whitehouse, M.E.A., Jaffe, K., 1996. Ant wars: combat strategies, territory and nest defence in the leaf cutting ant Atta laevigata. Animal Behavior 51, 12071217.

Wilson, E.O., 1971. The Insect Societies. Harvard University Press, Cambridge, MA

Wilson, E.O., 1978. Division of labor in fire ants based on physical castes (Hymenoptera: Formicidae: Solenopsis). Journal of the Kansas Entomological Society 51, 615636.

Wilson, E.O., 1980. Caste and division of labor in leaf cutter ants (Hymenoptera: Formicidae: Atta). I. The overall pattern in Atta sexdens. Behavioral Ecology and Sociobiology 7, 143156. 